\title{
OCORRÊNCIA DE PALINOMORFOS EM SEDIMENTOS PALEOZOIICOS DA BACIA DE JATOBÁ (PERNAMBUCO)
}

\author{
LUIZ PADILHA DE QUADROS*
}

\begin{abstract}
The palynological analysis of Paleozoic sediments of 2-IMst-1-PE (Ibimirim-Pernambuco, Jatobá Basin) well, yielded microfossils such Ancyrospora cf. ancyrea, Hystricosporites corystus, Maranhites brasiliensis, Reticulatisporites spp., Hymenozonotriletes lepidophytus, Knoxisporites spp. and Tholisporites sp. This association characterize the Upper Devonian (Strunian) and Lower Carboniferous (Tournaisian/Visean) to the interval studied.

On the Devonian sediments of Ibimirim well, were found a great amount of the genus Maranhites and Tasmanites which are normally associated with reducing conditions and sediments rich on radioative elements like uranium and radium.

The Devonian microfossils forms characterize marine conditions; on the Carboniferous section there are not evidences of the depositional environment.

The paper presents illustrations of the sporomorphs acompanied by correlation with Carboniferous and Devonian sediments from the Mac Mahon Basin (Algerian Sahara) and comparison with the Devonian from the Paraná Basin.
\end{abstract}

\section{INTRODUÇÃO}

A presença do paleozóico tem sido registrada em bacias sedimentares do nordeste do Brasil.

Na parte referente à palinologia do paleozóico, são raríssimos os trabalhos de ordem sistemática e zoneamento dos espécimes.

Neste estudo, apresenta-se a distribuição bioestratigráfica de esporomorfos encontrados no poço 2-IMst-1-PE (Ibimirim, Pernambuco - Bacia Jatobá) com tempo geológico entre o Devoniano até o Carbonífero.

Os esporomorfos estudados neste trabalho já haviam sido observados por Sommer (1964, comunicação pessoal) e por Regali (1964) no poço 2-IMst-1-PE (Fig. 1). Os referidos autores situam as amostras como pertencentes ao Carbonífero Inferior e Devoniano Médio.

$\mathrm{Na}$ presente revisão buscamos complementar as informações obtidas por Sommer (op. cit.) e Regali (op. cit:), visando fundamentalmente catalogar as formas-guia, correlacionando-as com padrões cronoestratigráficos mundiais.

No referente a litoestratigrafia, Souza e Cenachi (1964), em trabalho de semi-detalhe da Bacia de Jatobá, adotaram a subdivisão informal estabelecida por Almeida em 1962 onde os sedimentos do paleozóico foram designados pelas unidades $\mathrm{A}, \mathrm{B}, \mathrm{C}$ e $\mathrm{D}$. Na seção do poço Ibimirim, aparecem as unidades $C$ do Devoniano e D do Carbonífero. Barreto em 1968 formaliza essas unidades, designando a $\mathrm{C}$ de Formação Ibimirim e a $\mathrm{D}$ como Formação Moxotó, ambas pertencentes ao Grupo Jatobá ; observamos, no entanto, com base na datação dos sedimentos, que o contato

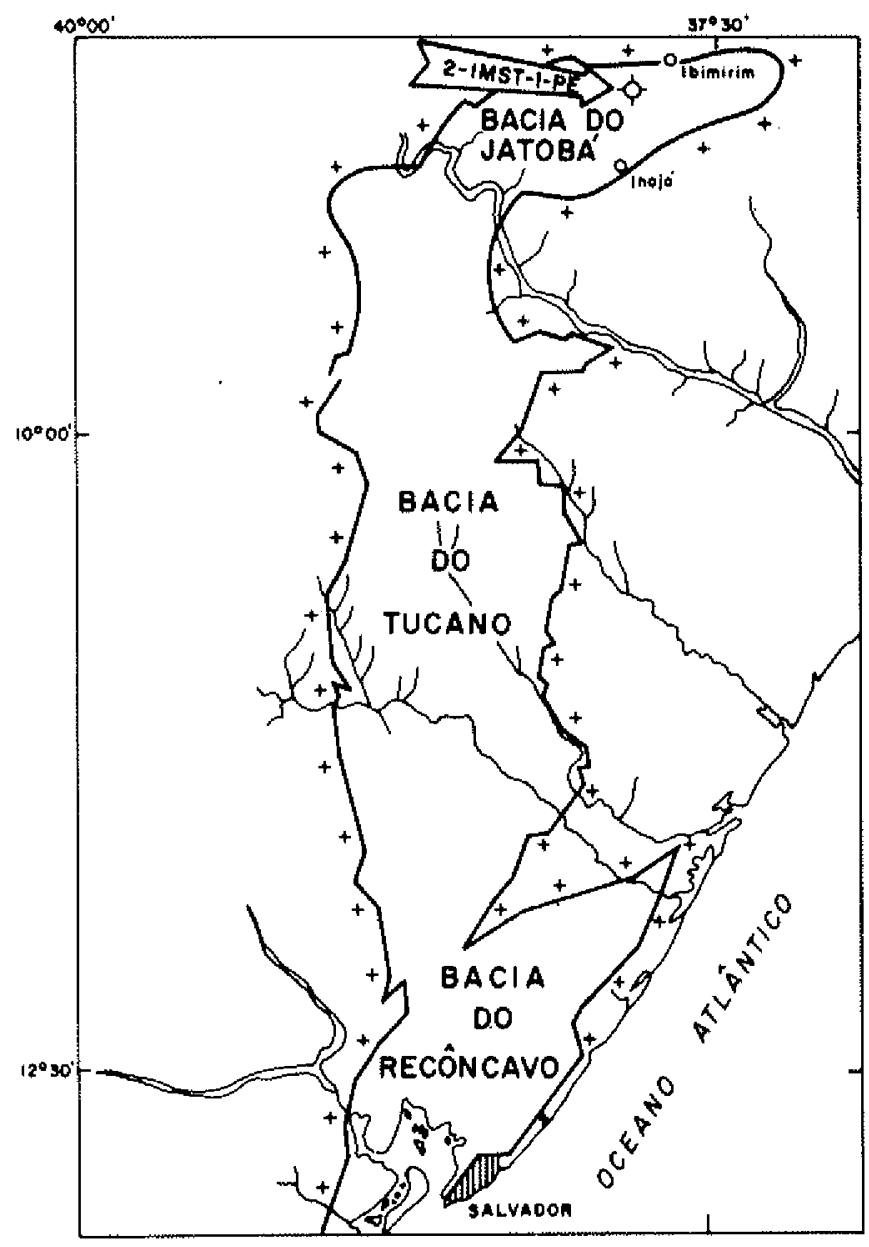

Figura 1 - Mapa de localização do poço 2-IMst-1-PE (Ibimirim, Pernambuco)

* Centro de Pesquisas e Desenvolvimento Leopoldo A. Miguez de Mello (CENPES), DIVEX - PETROBRÁS - Ilha do Fundão - Quadra 7 - Cidade Universitárja - 21910 - Rio de Janeiro - RJ. 
entre a Formação Ibimirim e a Formação Moxotó se dá aos 2652 metros e não aos 2675 metros como posicionado por Barretto (op. cit.).

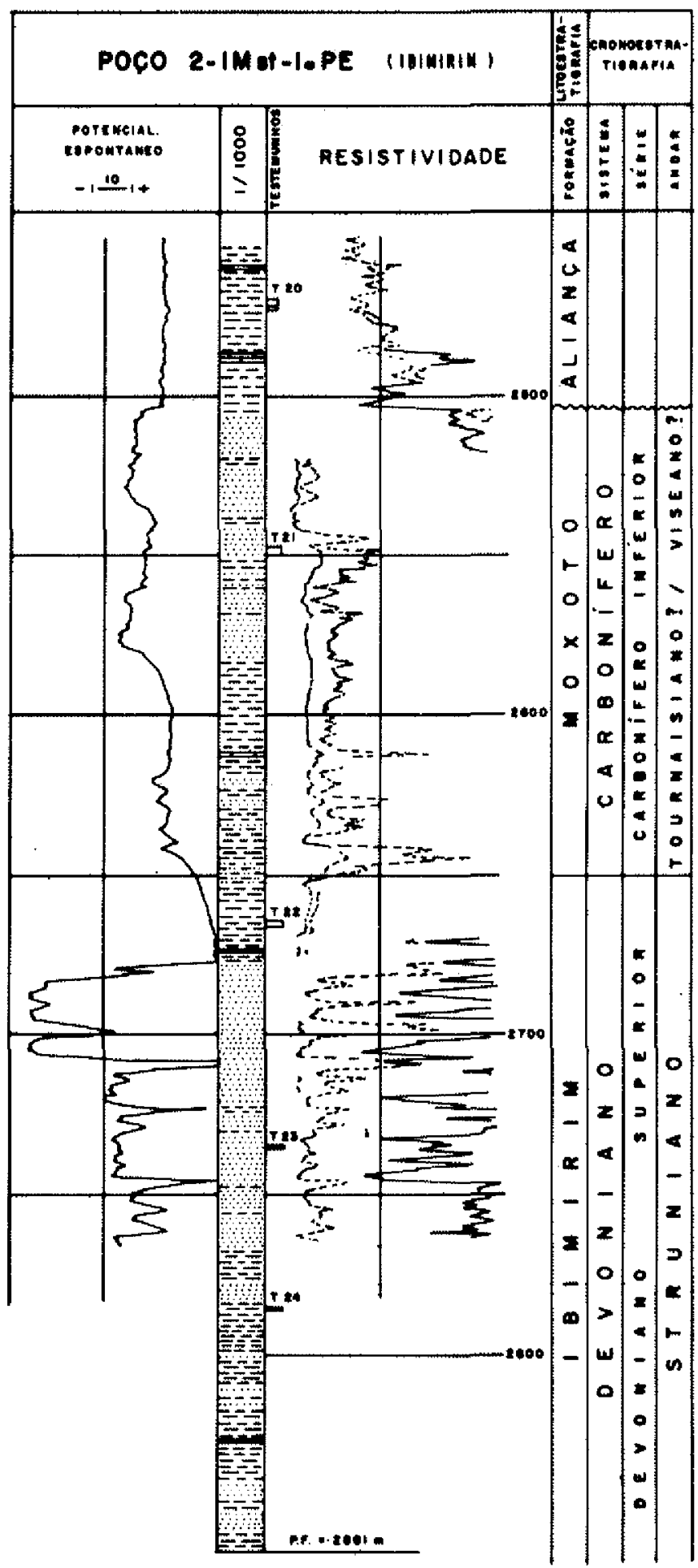

Figura 2 - Litoestratigrafia e cronoestratigrafia do poço 2-IMst-1-PE.
As amostras examinadas do poço pertencem ao intervalo 2550-2 730 metros, sendo 5 de calha e 1 de testemunho (Testemunho 22-2 663,8 metros).

Aos 2550 metros, encontrou-se a forma Tholisporites $\mathrm{sp} .1$ (E-608), considerada como pertencente ao Carbonifero Inferior (Estampa 1, Fig. 11). Na amostra de calha $2640-2670$ metros foram encontrados os primeiros indícios do Devoniano. De acordo com os dados bioestratigráficos, litologia e perfis elétricos, o topo do Devoniano se localiza aos 2652 metros. Dessa forma, o intervalo de 2535 até 2652 metros é caracterizado por uma associação de esporomorfos do Carbonífero Inferior (Tournaisiano/Viseano), e o intervalo 2652 a 2850 metros apresenta associação de espécimes do Devoniano Superior (Struniano).

SISTEMÁTICA DOS ESPOROMORFOS A sistemática dos esporomorfos se encontra em fase de contínua evolução. Existem numerosos sistemas de classificação, e a razão fundamental dessa diversidade reside no fato de que os esporomorfos raramente são encontrados in situ. $\mathrm{O}$ relacionamento seguro entre planta e esporo disperso, por vezes ou na maioria das vezes, é impossível. Dessa maneira, há necessidade de se utilizar um sistema artificial de classificação dos esporomorfos, onde os pesquisadores possam utilizálos em estudos bioestratigráficos.

De acordo com H. Potonié (1912) "se estuda a forma, sem preocupação com os pontos de vista teóri$\cos$, apenas para a catalogação prática".

R. Potonié $(1956,1958,1960)$ elaborou um sistema que obedece às regras do Código Internacional de Nomenclatura Botânica, classifica os fósseis quanto forma, facilitando o manuseio e identificação de novas formas. No presente estudo utilizaremos a classificação de R. Potonié (op. cit.). A nomenclatura dos espécimes sofre constante alteração, assim sendo, os Laboratórios da Petrobrás utilizam, além da classificação artificial de Potonié (op. cit.), um sistema particular de codificação das formas; os esporomorfos são designados pela letra $E$, seguida de um algarismo arábico. Sempre que possível, serão apresentadas as afinidades sistemáticas das diferentes formas.

PALEOECOLOGIA No intervalo 2652-2850 metros (Struniano), foram encontrados diversos espécimes de "Leiofusidae, Chitinozoa e Hystrichosphaeridade". Estas formas caracterizam ambiente marinho para o intervalo. Digno de nota é a abundância consisiderável de espécimes dos gêneros Maranhites e Tasmanites. De acordo com Combaz (1966), e conforme discutido por Daemon e Quadros (1970), os Tasmanites estão relacionados com sedimentos depositados em mejo redutor rico em elementos radioativos como urấnio e rádio.

Quanto a seção $2535-2652$ metros (provável Tournaisiano/Viseano), em virtude da pobreza em palinomorfos, são prematuras as considerações de ordem paleoecológica. 


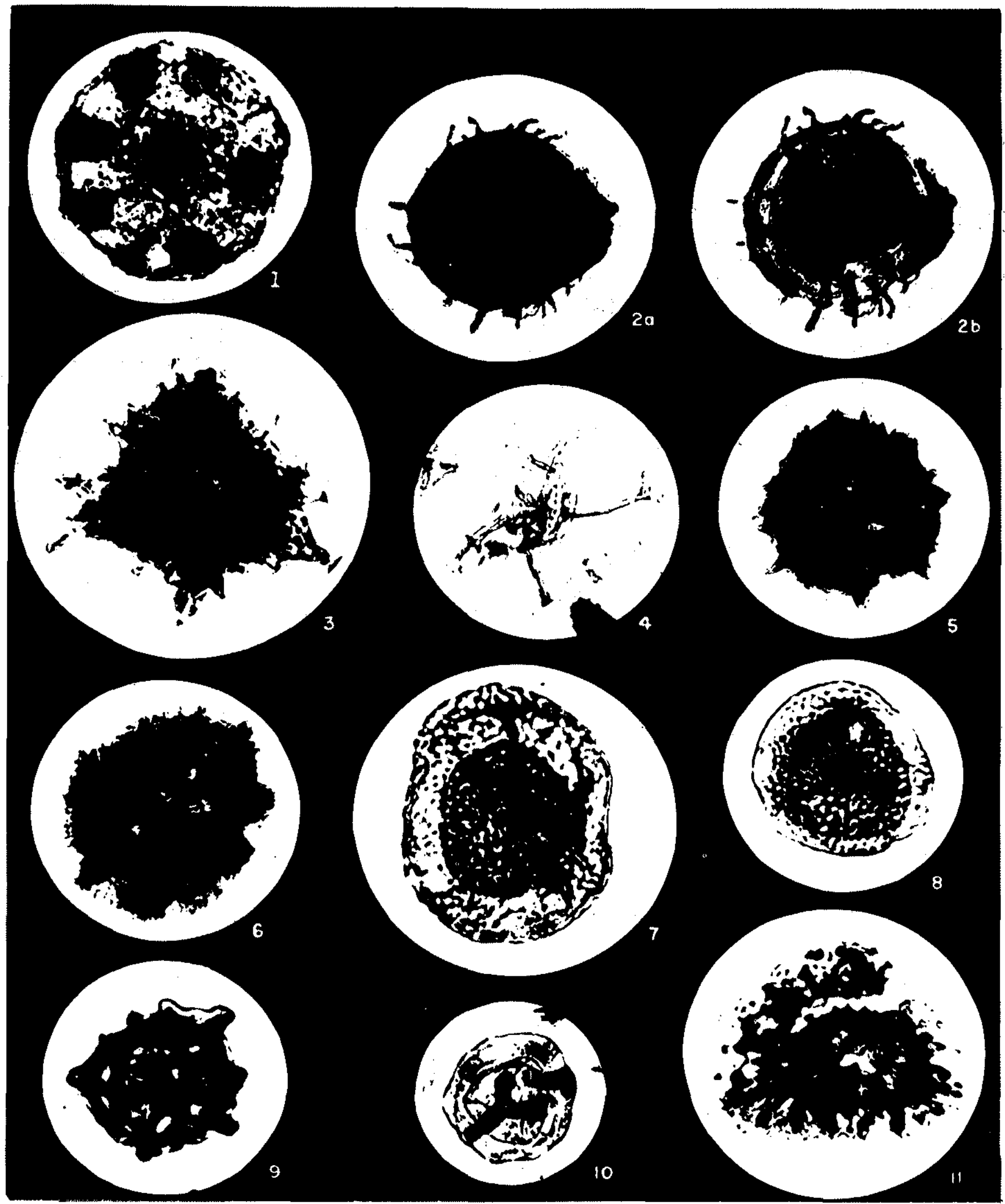

ESTAMPA 1

Fig. 1 - E-600; Lâmina 1, Poço 2-IMst-1-PE, calha 2 640-2 670 metros. Diâmetro da forma: $138 \mu$. Fig. 2a-2b-E-601; Lâmina 1, Poço 2-IMst-1-PE, calha $2640-2670$ metros. (2a) visão periférica. (2b) detalhe da espessura da exina. Diâmetro da forma sem apêndices: $243 \mu$. Fig. 3 - E-602; Lâmina 6; Poço 2-IMst-1-PE, Tèstemunho n. ${ }^{\circ} 22$ (2 663,8 metros). Diâmetro da forma: $140 \mu$. Fig. 4 - E-603; Lâmina 15, Poço 2-IMst-1-PE, Testemunho n. ${ }^{\circ} 22$ (2 663,8 metros). Diâmetro da forma com apêndices: $40 \mu$. Fig. 5-6 - E-604; Lâmina 1, Poço 2-IMst1-PE, calha $2640-2670$ metros. (5) Diâmetro da forma: $104 \mu$. (6) Diâmetro da forma: $111 \mu$. Fig. 7-8 - E-605; Lâmina 12, Poço 2-IMst1.PE, Testemunho n..$^{\circ} 22(2,663,8$ metros). (7) Diâmetros da forma: $49 \mu \times 65 \mu$. (8) Diâmetro da forma: $41 \mu$. Fig. 9 - E-606; Lâmina 11 , Poço 2-IMst-1-PE, calha $2670-2700$ metros. Diâmetro da forma: $62 \mu$. Fig. $10-$ E-607; Lâmina 13, Poço 2-IMst-1-PE, calha $2640-2670$ metros. Diâmetro da forma: $66 \mu$. Fig. 11 - E-608; Lâmina 2, Poço 2-IMst-1-PE, calha $2550-2580$ metros. Diâmetro da forma: $52 \mu$. 


$$
E-600
$$

Estampa 1, Figura 1

Taxinomia: Maranhites brasiliensis Forma L (Brito) Daemon, Quadros e Silva (1967)

De acordo com Daemon et alii (1967), o gênero $M a$ ranhites distribui-se na Bacia do Paraná do Givetiano ao Frasniano. Lanzoni e Magloire (1969) apresentam formas idênticas a Maranhites brasiliensis ocorrendo até o Tournaisiano. Conclui-se, portanto, que Maranhites brasiliensis se distribui do Givetiano ao Tournaisiano.

$$
\text { E- } 601
$$

Estampa 1, figuras 2a, b

Taxinomia: Hystricosporites corystus Richardsons, 1962.

Em trabalho sobre a Bacia do Baixo Amazonas. Daemon (1966) considera formas similares a $\boldsymbol{H}$. corystus como ocorrendo do Devoniano Médio ao Superior. Na Bacia do Paraná, Daemon et alii (1967) encontraram $H$. corystus somente no Frasniano. Richardson (1965) encontrou $\boldsymbol{H}$. corystus na Escócia como se distribuindo do Eifeliano ao Famenniano. No poço 2-IMst-1-PE se encontrou $H$. corystus associado a formas do Struniano.

$$
E-602
$$

Estampa 1, Figura 3

Taxinomia: Ancyrospora cf. ancyrea

As diversas variedades de Ancyrospora ancyrea, distribuem-se, de acordo com Richardson (1965), do Eifeliano ao Givetiano. Daemon et alii (1967) descrevem $A$. cf. ancyrea no Frasniano da Bacia do Paraná. No poço 2-IMst-1-PE se encontrou $A$. cf. ancyrea associada com formas do Struniano.

$$
E-603
$$

Estampa 1, Figura 4

Taxinomia: Grupo Acritarcha

E-603 foi registrada por Regali (1964, p. 176), em sedimentos devônicos do poço 2-IMst-1-PE. Lanzoni e Magloire $(1969$, Estampa 8, Figs. 17, 18) denominam informalmente este palinomorfo como "Acritarche à entonnoirs n." 441-33" e consideram-na como se distribuindo do Famenniano Superior ao Struniano.

$$
E-604
$$

Estampa 1, figuras 5 e 6

Taxinomia: Reticulatisporites sp. 1

Regali (1964, p. 179, Fig. 11, n. ${ }^{\circ} 6$ ) registrou essa forma no Devoniano do poço 2-IMst-1-PE, sob a designação de I-5. Lanzoni e Magloire (1969, Estampa 6, Figs. 7 e 8) apresentam espécimes similares a E-604 como do gênero Reticulatisporites sp. 3207 , com distribuição do Famenniano Superior do Tournaisiano.

$$
\text { E }-605
$$

Estampa 1, figuras 7 e 8

Taxinomia: Hymenozonotriletes lepidophytus Kedo 1957

Esta forma, de acordo com Streel (1967), é um guia que corresponde ao limite Devoniano/Carbonifero. Owens e Streel (1967) consideram $\boldsymbol{H}$. lepidophytus como um guia valioso na identificação dos depósitos do Famenniano ou Struniano. Esta forma foi registrada por Regali (1964, p. 170 , Fig. 2 n. ${ }^{\circ}$ ) sob a designação de Grupo T1-2 no poço 2-IMst-1-PE. Lanzoni e Magloire (1969) registraram $\boldsymbol{H}$. lepidophytus em depósitos do Famenniano/Struniano do Sahara Algeriano.

$$
E-606
$$

Estampa 1, Figura 9

Taxinomia: Knoxisporites sp. 1

A forma E-606 é similar ao espécime apresentado por Lanzoni e Magloire (1969, estampa 5, Figs. 3 e 4) sob a designação de Knoxisporites sp. 3286 e distribuem-se do Struniano ao Tournaisiano. Barss (1967) encontrou espécimes de Knoxisporites desde o Viseano até o Westphaliano do Canadá. Assim sendo, o gênero Knoxisporites pode ser encontrado desde o Struniano até o Westphaliano.

$$
\text { E }-607
$$

Estampa 1, Figura 10

Taxinomia: Knoxisporites sp. 2

Barss (1967, Estampa X, Fig. 11) registrou forma similar a esta no Viseano do Canadá, sob a designação de Knoxisporites hederatus. Lanzoni e Magloire (1969. Estampa 6, Figs. 5 e 6) apresentam formas similares a E-607, designando-as de Knoxisporites sp. n..$^{\circ} 2895$, considerada como se distribuindo do Famenniano Superior ao. Struniano.

$$
\mathrm{E}-608
$$

\begin{tabular}{|c|c|c|c|c|c|c|c|c|c|}
\hline IDADE & $\begin{array}{l}N \\
8 \\
0 \\
1 \\
u\end{array}$ & $\begin{array}{l}- \\
8 \\
0 \\
1 \\
w\end{array}$ & $\begin{array}{l}8 \\
8 \\
0 \\
1 \\
w\end{array}$ & $\begin{array}{l}m \\
0 \\
0 \\
1 \\
w\end{array}$ & $\begin{array}{l}\dot{8} \\
0 \\
1 \\
w\end{array}$ & $\begin{array}{l}8 \\
8 \\
1 \\
w\end{array}$ & $\begin{array}{l}\hat{8} \\
8 \\
1 \\
\omega\end{array}$ & $\begin{array}{l}0 \\
8 \\
1 \\
1 \\
w\end{array}$ & $\begin{array}{l}\text { : } \\
8 \\
\vdots \\
1\end{array}$ \\
\hline VISEANO & & & & & & & ? & & \\
\hline TOURNAISIANO & & & & & & 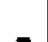 & 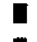 & & \\
\hline STRUNIANO & & & & & & & & & \\
\hline FAMENNIANO & & & & & & 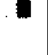 & 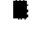 & & \\
\hline FRASNIANO & & & & & & & & & \\
\hline GIVETIANO & & & & & & & & & \\
\hline EIFELIANO & & & & & & & & & \\
\hline
\end{tabular}

Taxinomia: Tholisporites sp. 1

Hughes e Playford (1961) apresentam na Estampa 4, Figs. 1-7, diversas formas de Tholisporites foveolatus do Carbonifero Inferior de Spitsbergen. As formas E-608 assemelham-se a Tholisporites.

A distribuição bioestratigráfica mundial das principais formas encontradas no poço 2-IMst-1-PE é apresentada na Fig. 3.

\footnotetext{
E-602 AMCYROSPORA GF. AMCYRA (EISENACK) RICHAROSON 1962 E- 801 arstricospozires CORYSTUS RICHAROSON 1962

E- 600 MARAMHIES URASHIENSIS FORM I (BRITO) OAEMON, QUAOROS I SILVA 196 ?

E - $603 \approx$ ACRTARCHA $n$ E 44.33 LANZONI a MAGLOHE 1969

E-604 EFICULATISPONITS SP. Ex R.SP. J207 LANZONI MAGLOIRE 1969

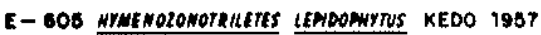

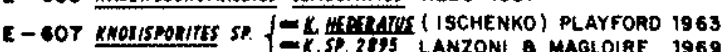
-

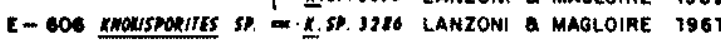

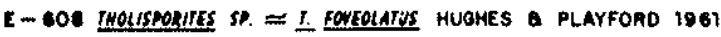

Figura 3 - Distribuição bioestratigráfica mundial dos principais esporomorfos do poço 2-IMst-1-PE. 
CORRELAÇÕES A rica associação de esporomorfos do intervalo 2652-2850 metros do poço 2IMst-1-PE, permitiu compará-la com o zoneamento estabelecido por Lanzoni e Magloire (1969), em sedimentos do Devoniano Superior/Carbonífero Inferior do Sahara Algeriano (Fig. 4). Esses autores apresentam várias zonas palinológicas, sendo que o intervalo L9 e L10 correspondentes ao Struniano são equivalentes ao intervalo 2652-2 850 metros do poço 2-IMst1-PE. Na comparação dos sedimentos do.Devoniano do poço 2-IMst-1-PE com os da Bacia do Paraná, onde os intervalos bioestratigráficos estão bem definidos, verifica-se que na área de Ibimirim os intervalos bioestratigráficos são mais novos (Fig. 4).

O intervalo $2535-2652$ metros pertence, no poço 2-IMst-1-PE, ao Carbonífero Inferior (Tournaisiano/ Viseano).

A coleta de amostras de superfície, em seções estratigráficas bem definidas da Bacia de Jatobá, poderia contribuir para a elaboração da reconstituição geocronológica da área. Estes resultados deverão auxiliar num melhor entendimento das ligações pretéritas entre as diversas bacias paleozóicas do Brasil.

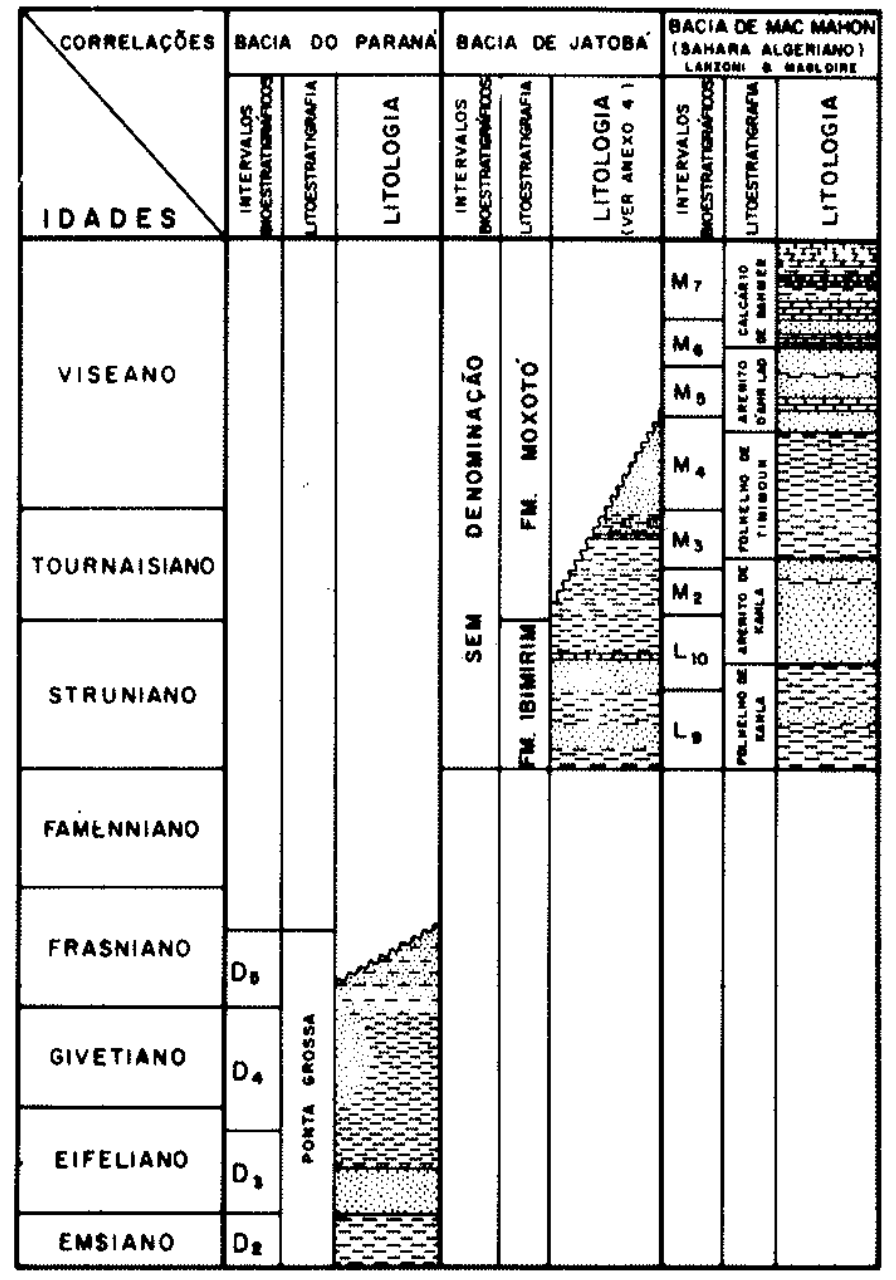

Figura 4 - Correlação bioestratigráfica do poço 2-IMst-1PE com a Bacia de Mac Mahon (Sahara algeriano) e comparação com a Bacia do Paraná.

\section{BIBLIOGRAFIA}

ALMEIDA, A.C.S. - 1962 - Viagem de reconhecimento às bacias de Tucano Norte e Jatobá, Rel SETEX, RPBA, n.0 533.

BARRETO, P.M.C. - 1968 - O Paleozóico da Bacia de Jatobá, Pernambuco. Bol. Soc. Bras. Geol. 17(1), 29-45.

BARSS, M. S. - 1967 - Carboniferous and Permian Spores of Canada. Geol. Serv. Can. Paper 67-11.

COMBAZ, A. - 1966-Remarque sur les niveaux a Tasmanacées du Paléozoique Saharien. Palaeobotanist, v. 15, n. ${ }^{\circ}$ 1. 2, pl. 1-2, 29-34.

DAEMON, R. F. - 1966 - Contribuição ao estudo dos esporomorfos siluro-devonianos da Bacia do Baixo Amazonas. SETEX/DESUL, Rel. 342.

DAEMON, R. F. \& QUADROS, L. P. - 1970 - Biocstratigratia do Neopaleozóico da Bacia do Paraná. Anais XXIV Congr. Bras. Geol. Soc. Bras. Geol., Brasilia, 359.412.

DAEMON R. F., QUADROS, L. P. \& SILVA, L. C. - 1967 - Devonian Palynology and Biostratigraphy of the Paraná Basin. Bol. Paran. Geoc. $n .^{\circ} 21-22,99-132$

HUGHES, N. F. \& PLAYFORD, G. - 1961 - Palynological Reconaissance of the Lower Carbonilerous of Spitsbergen. Micropaleontology. v. $7, n .{ }^{\circ} 1,27.44$

KEDO, G. I. - 1957 - On the stratigraphy and spore pollen complexes of the lower horizons of the Carbonilerous in the BSSR: Dokl. Akad. Navk. SSSR, n."115, 1165-1168.

LANZONI, E. \& MAGLOIRE, L. - 1969 - Associations palynologiques et leurs applications stratigraphiques dans te Dévonien Supérieur et Carbonifére Inférieur du Grand Erg Occidental (Sahara Algérien). Revue de L'Institut Français du Pétrole, v. 24, n. ${ }^{\circ} 4,441-469$.
OWENS, B. \& STREEL, M. - $1967-$ Hymenozonotriletes lepidophytus KEDO its distribution and significance in relation to the DevonianCarboniferous Boundary. Rev. Palaeobotan. Palynol. v. 1, 141-150.

POTONIÉ, H. - 1912 - Grundlinien der Pflanzenmorphologie im Lichte der Palaeontologie. Jena (G. Fischer).

POTONIE, R. - 1956 - Synopsis der Gattungen der Sporae Dispersae I: Hannover, Beih, Geol., Jb., 23, 103 p.

POTONIÉ, R. - 1958 - Synopsis der Gattungen der Sporae Dispersae II Hannover, Beih. Geol. Jb. 31, 1-114

POTONIE, R. - 1960-Synopsis der Gattungen der Sporae Dispersae III. Hannover Geol. Jb, 39, 1-189.

REGALI, M. S. P. - 1964 - Resultados palinológicos de amostras Paleozóicas da Bacia Tucano-Jatobá. Bol. Téc. Petrobrás 7 (2), 132-282.

RICHARDSON, J. B. $-1962-$ Spores with bifurcate processes from the Middle Old Red Sandstone of Scotland. Palaeontology 5, 171-194.

RICHARDSON, J. B. - 1965 - Middle Old Red Sandstone Spores assemblages from the Orcadian Basin - North East Scolland. Palaeontology $7,559-603$.

SOMMER, F. W. - 1959 - Introdução ao estudo sistemático dos gêneros paleozóicos de esporos dispersos Il - Pollenites. DNPM, Div. Geol. Min., Rio de Janeiro, Bol. 190, 1:221.

SOUZA, O. R, \& CENACHI, N. C. $-1964-$ Semi-detalhe geológico da Bacia de Jatobá, RPBA, Rel. 740.

STREEL, M. - 1967 - Associations de spores des Stratotypes du Famennien, du Strunien et du Tournaisien dans les Bassins Ardeno-Rhénans (Note Préliminaire). Rev. Palaeobotan. Palynol. v. 5, 63-74. 